\title{
Short-term prediction of preeclampsia: how close are we?
}

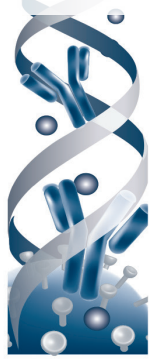

"Currently, delivery is the only cure, and preeclampsia remains the leading reason for iatrogenic preterm delivery."

Keywords: angiogenic $\bullet$ biomarker $\bullet$ hypertension $\bullet$ prediction $\bullet$ preeclampsia $\bullet$ pregnancy - test performance

Preeclampsia is a disease of pregnancy that contributes substantially to maternal and perinatal morbidity and mortality around the globe, with 60,000 maternal deaths attributed to hypertensive disorders of pregnancy every year [1]. Currently, delivery is the only cure, and preeclampsia remains the leading reason for iatrogenic preterm delivery [2]. Preterm preeclampsia (requiring delivery before 37 weeks' gestation) is associated with greater complications for mother and infant, compared with disease at term. Current antenatal care throughout the world is directed towards identification of the end points of the disease (through measurement of blood pressure and urinary protein estimation) but this approach lacks accuracy, and women with suspected disease are often overmanaged or neglected. Reports have demonstrated substandard care related to misinterpretation of current assessment [3].

Accurate prediction of preeclampsia could occur at several time points: in the first half of pregnancy; at a time point remote from the disease but with maximal opportunity to instigate prophylactic measures; and in the third trimester when women present with suspected preeclampsia. This latter scenario produces a common management dilemma for most maternity clinicians, with optimal care depending on accurate identification of those with clinically relevant disease. Preeclampsia is not a uniform entity; its heterogeneity encompasses multiple presentations of maternal disease (in which dysfunction of one organ system may predominate, e.g., severe hypertension, acute kidney injury, hepatic dysfunction, clotting abnormalities or seizures) and with varying degrees of fetal disease usually marked by growth restriction. The onset at which preeclampsia first presents may vary from late second trimester ( 24 weeks of pregnancy) through to presentation beyond term (e.g., past 40 weeks) or even with first onset in the postnatal period. Given the diversity of this clinical condition it is unsurprising that there is no reliable remote prediction of preeclampsia in routine clinical practice and even short-term prediction remains challenging.

\section{What are the benefits of short-term prediction?}

Short-term prediction seeks to identify those women who require an increase in their surveillance and/or possible therapeutic intervention to ameliorate the adverse consequences of the disease; it is also beneficial if a test can accurately rule out those who do not have the disease. Symptoms cannot be relied on for risk assessment as there is considerable overlap between those of normal pregnancy (e.g., headache, epigastric pain and edema) and those associated with preeclampsia. Measurement of both hypertension and proteinuria is fraught with measurement and technical challenges, with false-positive and negative assessments common, and an impre-

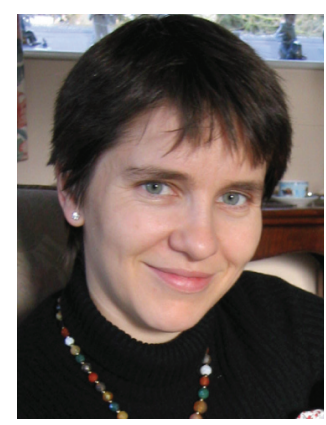

Lucy C Chappell Author for correspondence: Women's Health Academic Centre, King's College London, London, SE1 7EH, UK

Tel.: +442071883639

Fax: +44 2076201227

lucy.chappell@kcl.ac.uk

Kate Bramham

Women's Health Academic Centre, King's College London, London, SE1 7EH, UK

Andrew H Shennan Women's Health Academic Centre, King's College London, London, SE1 7EH, UK

Future $\because \%$ Medicine part of 
cise relationship to adverse outcome. Other authors have proposed that a predictive test for preeclampsia should have high sensitivity and high negative predictive value to be most useful [4]; this would enable identification of the great majority of cases, minimization of false negatives and parallel recognition of women with normal biomarker values who could return to routine care.

\section{What are the harms of prediction?}

Predictive tests also have harms. Any test with false positives can induce anxiety (and false negatives can induce false reassurance). If doctors act on a predictive test for preeclampsia inappropriately, there is the very real potential to cause substantial morbidity through iatrogenic premature delivery of an infant. It is essential that a novel biomarker test has adequate test performance to minimize such harms, and that the implications of a positive result for consequent management are also considered.

\section{What biomarkers do we currently use to predict preeclampsia?}

In women presenting with suspected preeclampsia in the third trimester of pregnancy, current practice relies on blood pressure and proteinuria reaching threshold values to confirm or refute the diagnosis. This definition has revolved around identification of two clinical manifestations of the disease that were most easily measured to the physicians of the early 20th century, rather than on strong pathophysiological evidence. Additional laboratory tests commonly used to investigate end-organ damage of preeclampsia include maternal platelet count, liver transaminase or uric acid concentrations; however, systematic reviews have consistently shown that quantification of proteinuria, uric acid or transaminases do not have high sensitivity for prediction of need for delivery or maternal or fetal complications of the disease [5-7].

\section{"Short-term prediction seeks to identify those women who require an increase in their surveillance and/or possible therapeutic intervention to ameliorate the adverse consequences of the disease."}

The pathophysiological process starts with abnormal placentation in the first trimester of pregnancy. In some pregnancies, there is a failure of normal spiral artery remodelling, which results in ongoing high pressure, pulsatile flow rather than normal low pressure flow; subsequent placental oxidative stress leads to release of factors into the maternal circulation [8]. The most promising biomarkers that reflect this placental dysfunction are PlGF and sflt-1. PIGF is an angiogenic factor produced principally by trophoblast cells. PlGF interacts with cell surface receptors such as flt-1; this receptor also exists in soluble form ( $(\mathrm{sflt}-1)$, which binds PIGF in the maternal circulation. Modern assays measure free PlGF, not bound to sft-1. In normal healthy pregnancies, maternal concentrations of PlGF peak at approximately 28-30 weeks gestation and then fall towards term whereas in women with preeclampsia, slft- 1 is upregulated, and PlGF is decreased in the maternal circulation [9].

\section{“Approximately $10-15 \%$ of all pregnant women develop gestational hypertension and many more may have transient symptoms ... that require evaluation, but only $5 \%$ develop confirmed preeclampsia."}

Interest has, therefore, moved from the description of altered biomarker concentrations in established disease to their application for short-term prediction in women presenting with suspected preeclampsia. The aim of this approach is to target management more appropriately, resulting in reduced maternal and neonatal morbidity and preventing unnecessary admissions and investigations. Approximately $10-15 \%$ of all pregnant women develop gestational hypertension and many more may have transient symptoms (including headache, epigastric pain and visual symptoms) that require evaluation, but only $5 \%$ develop confirmed preeclampsia.

In a single-center study of 176 women presenting with suspected preeclampsia before 35 weeks gestation, Rana and colleagues reported that a threshold of 85 for the sft-1/PlGF ratio (using the Elecsys platform, Roche, Penzburg, Germany) had sensitivity of $73 \%$ and specificity of $95 \%$ for predicting maternal adverse outcome [10]. A subsequent multicentre study of 287 women with similar inclusion criteria assessed PIGF using a different assay (Triage test, Alere, CA, USA); low maternal PIGF concentrations ( $<$ fifth centile) had very high sensitivity (96\%) and negative predictive values $(98 \%)$ for determination of preeclampsia requiring delivery within 2 weeks [11]. When compared with currently utilized tests, the area under the receiver operating characteristic curve for low PlGF (0.87, standard error 0.03 ) for predicting preeclampsia within 14 days was greater than all other commonly used tests, either singly (systolic blood pressure: 0.67 [0.05]; diastolic blood pressure: 0.66 [0.05]; uric acid: 0.68 [0.06]; alanine transaminase: 0.61 [0.05]; dipstick proteinuria: 0.76 [0.04]) or in combination (0.70 [0.05]; p $<0.001$ for all comparisons). A single test could supersede all other clinical evaluations combined. Although masked at the time of the study, low (<fifth centile), 
maternal PlGF concentrations were found in all seven cases of antepartum stillbirth in this cohort, often weeks before abnormal ultrasound findings, suggesting that appropriate fetal surveillance would have allowed timely intervention. This cohort study confirms a number of case-control studies that had previously reported promising performance.

Other biomarkers have been extensively studied, through both systematic reviews [12] and consensus meetings [13]. The list of candidates includes angiogenic factors, as described above, and other molecules from the fetoplacental unit (e.g., $\alpha$-fetoprotein, human chorionic gonatotrophin, pregnancy associated plasma protein-A and placental protein 13), cell-free fetal DNA and RNA, markers of maternal origin (e.g., reflecting alterations in the renin-angiotensin system or proinflammatory markers such as pentraxin) and biophysical markers, including blood pressure and uterine artery Doppler flow velocity waveforms. Many of these biomarkers have emerged from case-control studies where women with preeclampsia have been compared with those with normal healthy pregnancies. In clinical practice, a biomarker needs to discriminate those with preeclampsia from all other diagnoses (e.g., gestational hypertension). Thus initial estimates of biomarker performance are usually overly optimistic when compared with subsequent rigorous testing in prospective multicenter cohort studies.

\section{What are the clinical implications?}

The test performance reported for PIGF in women presenting with suspected preeclampsia has been confirmed in further similar cohort studies [14] and in others assessing prediction of preeclampsia, stillbirth and fetal growth restriction using a maternal blood sample at a fixed time point of 30-34 weeks gestation [15]. With suspected preeclampsia the commonest reason for presentation in the third trimester for urgent obstetric review, these studies suggest that adding PIGF measurement to current clinical assessment could improve risk stratification, enabling an earlier diagnosis to be reached such that those at greatest risk receive appropriate surveillance, while those at lower risk can avoid unnecessary admissions and intervention.

\section{What are the cost implications?}

A recent decision-analytic modeling analysis estimated that there could be a US $\$ 1400$ cost saving

\section{References}

1 Bhutta ZA, Chopra M, Axelson H et al. Countdown to 2015 decade report (2000-2010): taking stock of maternal, newborn, and child survival. Lancet 375(9730), 2032-2044 (2010). associated with the introduction of angiogenic factor (slft-1/PlGF ratio) testing in pregnant women for early diagnosis of preeclampsia [16]. Future developments, including adaptation of the platform for whole blood point of care testing, improves the potential for both clinical utility and increased cost savings further.

\section{What are the next steps?}

Obstetric units around the world are now considering introduction of PIGF, or sftt-1/ PlGF ratio, for use in clinical practice in women presenting with suspected preeclampsia. The ideal scenario would be to evaluate the test in a randomized controlled trial (e.g., of revealed vs concealed result), where comprehensive implementation of a test is considered, including consideration of downstream changes in patient management that result from the test [17]. There is also growing interest in using such tests in the most diagnostically challenging group of pregnant women with chronic hypertension and pre-existing renal disease (in whom identification of superimposed preeclampsia currently requires somewhat arbitrary increases of blood pressure and proteinuria or creatinine to reach a diagnosis). Preliminary results have demonstrated that PlGF also has high test performance in these populations [18].

The advent of a biomarker with such impressive test performance raises the possibility of revolutionizing antenatal management, particularly through point-of-care testing for short-term prediction of preeclampsia in women presenting with suspected disease. Use of an objective biomarker for defining women with preeclampsia and underlying placental dysfunction could identify women with adverse perinatal outcomes, provide a tighter research definition for comparison of interventions across populations [19] and potentially enable development of targeted therapeutic strategies.

\section{Financial \& competing interests disclosure}

AH Shennan has has been paid as a consultant for Alere, Roche and Perkin Elmer. The authors have no other relevant affiliations or financial involvement with any organization or entity with a financial interest in or financial conflict with the subject matter or materials discussed in the manuscript apart from those disclosed.

No writing assistance was utilized in the production of this manuscript.

2 Meis PJ, Goldenberg RL, Mercer BM et al. The preterm prediction study: risk factors for indicated preterm births. Maternal-Fetal Medicine Units Network of the National Institute of Child Health and Human Development. Am. J. Obstet. Gynecol. 178(3), 562-567 (1998). 
3 Shennan AH, Redman C, Cooper C, Milne F. Are most maternal deaths from pre-eclampsia avoidable? Lancet 379(9827), 1686-1687 (2012).

4 Cnossen JS, ter Riet G, Mol BW et al. Are tests for predicting pre-eclampsia good enough to make screening viable? A review of reviews and critical appraisal. Acta Obstet. Gynecol. Scand. 88(7), 758-765 (2009).

5 Thangaratinam S, Ismail KM, Sharp S, Coomarasamy A, Khan KS. Accuracy of serum uric acid in predicting complications of pre-eclampsia: a systematic review. BJOG 113(4), 369-378 (2006).

6 Thangaratinam S, Coomarasamy A, O'Mahony $\mathrm{F}$ et al. Estimation of proteinuria as a predictor of complications of pre-eclampsia: a systematic review. BMC Med. 7, 10 (2009).

7 Thangaratinam S, Koopmans CM, Iyengar S et al. Accuracy of liver function tests for predicting adverse maternal and fetal outcomes in women with preeclampsia: a systematic review. Acta Obstet. Gynecol. Scand. 90(6), 574-585 (2011).

8 Burton GJ, Woods AW, Jauniaux E, Kingdom JC. Rheological and physiological consequences of conversion of the maternal spiral arteries for uteroplacental blood flow during human pregnancy. Placenta 30 (6), 473-482 (2009).

9 Levine RJ, Maynard SE, Qian C et al. Circulating angiogenic factors and the risk of preeclampsia. N. Engl. J. Med. 350 (7), 672-683 (2004)

10 Rana S, Powe CE, Salahuddin S et al. Angiogenic factors and the risk of adverse outcomes in women with suspected preeclampsia. Circulation 125(7), 911-919 (2012).

11 Chappell LC, Duckworth S, Seed PT et al. Diagnostic accuracy of placental growth factor in women with suspected preeclampsia: a prospective multicenter study. Circulation 128(19), 2121-2131 (2013).
12 Hui D, Okun N, Murphy K, Kingdom J, Uleryk E, Shah PS. Combinations of maternal serum markers to predict preeclampsia, small for gestational age, and stillbirth: a systematic review. J. Obstet. Gynaecol. Can. 34(2), 142-153 (2012)

13 Cetin I, Huppertz B, Burton G et al. Pregenesys pre-eclampsia markers consensus meeting: What do we require from markers, risk assessment and model systems to tailor preventive strategies? Placenta 32(Suppl.), S4-S16 (2011).

14 Sibiude J, Guibourdenche J, Dionne MD et al. Placental growth factor for the prediction of adverse outcomes in patients with suspected preeclampsia or intrauterine growth restriction. PLoS ONE 7(11), e50208 (2012).

15 Chaiworapongsa T, Romero R, Korzeniewski SJ et al. Maternal plasma concentrations of angiogenic/antiangiogenic factors in the third trimester of pregnancy to identify the patient at risk for stillbirth at or near term and severe late preeclampsia. Am. J. Obstet. Gynecol. 208(4), 287 e1-e15 (2013).

16 Hadker N, Garg S, Costanzo C et al. Financial impact of a novel pre-eclampsia diagnostic test versus standard practice: a decision-analytic modeling analysis from a UK healthcare payer perspective. J. Med. Econ. 13(4), 728-737 (2010).

17 Ferrante di Ruffano L, Hyde CJ, McCaffery KJ, Bossuyt PM, Deeks JJ. Assessing the value of diagnostic tests: a framework for designing and evaluating trials. BMJ 344, e686 (2012).

18 Bramham K, Seed PT, Nelson-Piercy C et al. Diagnostic accuracy of placental growth factor in women with chronic kidney disease and suspected preeclampsia: a prospective cohort study. J. Am. Soc. Nephrol. 24, 142A (2013).

19 Staff AC, Benton SJ, von Dadelszen P et al. Redefining preeclampsia using placenta-derived biomarkers. Hypertension 61(5), 932-942 (2013). 